\title{
Falencias metodológicas y estadísticas en la valoración diagnóstica imagenológica: Precisiones y recomendaciones
}

Methodological and statistical flaws in diagnostic imaging evaluation: Precisions and recommendations.

\section{Sr. Editor:}

Hemos leído el artículo "Concordancia entre la radiografía panorámica y la tomografía computarizada de haz cónico en la relación de los terceros molares mandibulares con el conducto dentario inferior" de Herrera et al.,(1); el cual, aunque consideramos un valioso aporte a la evidencia local, presenta serias deficiencias metodológicas y estadísticas que afectan negativamente su calidad.

El primer problema que identificamos es que los autores se han centrado solamente en la concordancia, lo que brinda una perspectiva limitada y desaprovecha una valiosa oportunidad para obtener data local sobre medidas diagnósticas relevantes en la práctica clínica y toma de decisión; tales como la sensibilidad y especificidad diagnóstica $(94,5$ y 41,0\%), el valor predictivo positivo y negativo $(75,0$ y $80 \%)$ entre otros (2).

El segundo aspecto observado son los vacíos en la redacción (3); p.e. no se explicita cómo se llego al cálculo muestral de 62 participantes y de qué forma se seleccionaron, si el estudio fue muestral o censal, o los sesgos inherentes a ello. Así mismo, los resultados se presentan de forma desordenada, no incluyen información resaltante del estudio (p.e. los valores p), y confunden términos como "correlación" y "asociación estadística".

Entre las falencias metodológicas destaca que se seleccionaron sujetos con diverso grado de desarrollo radicular, lo que genera sesgo en los resultados, y podría haberse corregido mediante un análisis de subgrupos (4). Por otro lado, no existen datos claros sobre la cantidad de muestra empleada en la calibración; o si se ejecutó también a nivel intra-examinador, recomendable en estudios imagenológicos.

Finalmente, a nivel estadístico, es donde se pueden evidenciar las debilidades y errores más grandes del artículo, ya que en el plan de análisis se hace mención al uso de pruebas como Chi cuadrado, U de Mann Whitney y Kruskall Wallis; las que no guardan coherencia con el objetivo del estudio, y que anecdóticamente, no podemos encontrar que hayan sido utilizadas. Así, además los autores, erróneamente señalan: "El presente estudio contó con un $\mathrm{p}<0.05$ "; cuando el valor de $\mathrm{p}$ es calculado por la prueba estadística, mientras que lo que si definen los investigadores es el "nivel de significancia" (5). En cuanto al análisis de concordancia, podemos identificar que solo se expresa el valor del índice 
Kappa de Cohen $(0,4010)$, sin que se presente el p-valor correspondiente $(<0,001)$, ni mucho menos los intervalos de confianza al 95\% (0,2276; 0,5743). Adicionalmente, el artículo no menciona el potencial conflicto de interés existente, ni la fuente de financiamiento, requisitos generales de su revista. En conclusión, pese a su relevancia, la validez y calidad del artículo es cuestionable, por lo que no aporta evidencia solida para la toma de decisiones en el ámbito clínico.

Financiamiento: El presente manuscrito fue elaborado sin ningún tipo de financiamiento.

Conflicto de Interés: Los autores declaran no poseer ningún tipo de conflicto de interés.

\section{Gilmer Solis ${ }^{1, a}$, Yolanda Angulo-Bazán ${ }^{2, b}$}

\section{Correspondencia:}

Gilmer Solis Sánchez

Correo electrónico: remlig@outlook.com

\section{REFERENCIAS BIBLIOGRÁFICAS}

1 Herrera RR, Rios LK, León RA, Beltrán JA. Concordancia entre la radiografía panorámica y la tomografía computarizada de haz cónico en la relación de los terceros molares mandibulares con el conducto dentario inferior. Rev Estomatol Herediana. 2020;30(2):86-3.

2. Lima TF, Gamba TO, Zaia AA, Soares AJ. Evaluation of cone beam computed tomography and periapical radiography in the diagnosis of root resorption. Aust Dent J. 2016;61(4):425-31.

3. Sarkis-Onofre R, Cenci MS, Demarco $\mathrm{FF}$, et al. Use of guidelines to improve the quality and transparency of reporting oral health research. J Dent. 2015;43(4):397-04.

4. Sun X, Ioannidis JP, Agoritsas T, Alba AC, Guyatt G. How to use a subgroup analysis: users' guide to the medical literature. JAMA. 2014;311(4):405-411.

5. Andrade $\mathrm{C}$. The $\mathrm{P}$ value and statistical significance: misunderstandings, explanations, challenges, and alternatives. Indian J Psychol Med. 2019;41:210-5.

Recibido: 13-07-20

Aceptado: 18-09-20 